\title{
Effect of Methyl Paraben on Sludge Reduction
}

\author{
Guo Wangqian, Chen Tianhui \\ Environmental School, Harbin Institute of Technology, Haerbin, China
}

\section{Email address:}

guowanqian@hit.edu.cn (Guo Wangqian),18745067687@163.com (Chen Tianhui)

\section{To cite this article:}

Guo Wangqian, Chen Tianhui. Effect of Methyl Paraben on Sludge Reduction. Science Discovery. Vol. 6, No. 3, 2018 , pp. 175-183. doi: 10.11648/j.sd.20180603.16

Received: April 4, 2018; Accepted: May 8, 2018; Published: June 23, 2018

\begin{abstract}
Activated sludge process is the most widely used sewage treatment technology, which accounts for more than $90 \%$ of urban sewage treatment processes and about $50 \%$ of industrial wastewater treatment processes. The essence of the activated sludge process is that microorganisms use the biodegradable organic matter in the sewage as nutrients for metabolism and meanwhile remove them, while large amounts of sludge would be generated inevitably during the sewage treatment process. The cost for the sludge treatment accounts for about $50 \%$ of the operating cost of the sewage treatment plant. In this study, the effects of uncoupler propyl paraben on sludge reduction and sewage treatment were conducted, and the results showed that the optimized concentration of propyl paraben for sludge reduction was $30 \mathrm{mg} / \mathrm{l}$, which achieved $41 \%$ sludge reduction effect. Under the optimized propyl paraben concentration, the average removal rate of COD increased from $93.35 \%$ in the blank group to $94.53 \%$; the average removal rate of $\mathrm{NH}_{4}{ }^{+} \mathrm{N}$ increased from $65.47 \%$ in the blank group to $83.84 \%$; the average removal rate of $\mathrm{PO}_{4}{ }^{3-}$-P from the $58.47 \%$ in the blank group increased to $67.36 \%$; meanthile the sludge kept good settalbility during the sewage treatment process.
\end{abstract}

Keywords: Propyl Paraben, Sewage Treatment, Sludge Reduction

\section{尼泊金丙酯对污泥减量的效果研究}

\author{
郭婉茜, 陈田慧 \\ 环境学院, 哈尔滨工业大学, 哈尔滨, 中国
}

\section{邮箱}

guowanqian@hit. edu. cn (郭婉茜)，18745067687@163. com(陈田慧)

摘要: 活性污泥法是目前应用最为广泛的污水处理技术，占城市污水处理工艺的 $90 \%$ 以上和工业废水处理工艺的 50\%左右。活性污泥法的本质是微生物利用污水中可生物降解的有机物作为营养物质进行代谢活动并将其去除, 该方法在处理污水的同时不可避免的会产生大量生物污泥。污泥的处理费用占污水处理厂运行成本的 $50 \%$ 左右。 本实验研究了解偶联剂尼泊金丙酯对污泥减量及污水处理效果的影响。研究发现, 尼泊金丙酯的浓度为 $50 \mathrm{mg} / \mathrm{L}$ 时, 污泥的减量效果最佳达到了 $47 \%$ 。此条件下, COD 的平均去除率由空白组的 93. 35\%提高到 $94.53 \%$; $\mathrm{NH}_{4}{ }^{+}-\mathrm{N}$ 的 平均去除率由空白组的 $65.47 \%$ 提高到 $83.84 \%$; $\mathrm{PO}_{4}{ }^{3-}-\mathrm{P}$ 的平均去除率会从空白组的 $58.47 \%$ 提高到 $67.36 \%$; 在水处 理工程中, 污泥保持良好的沉降性能。

关键词: 尼泊金丙酯, 污水处理, 污泥减量 


\section{1. 引言}

目前污水处理的方法很多, 活性污泥法是应用最广泛 的污水处理方法 [1]。进行污水处理的同时, 不可避免的会 产生大量的剩余污泥。文献表明, 对这部分污泥的处理费 用占污水处理厂总运行费用的 $40 \%-60 \%$ 。目前污泥减量主 要有两种方法: 污泥后减量和污泥的过程减量。

污泥后减量: 污泥中含有大量的病原体、寄生虫、有 机污染物, 重金属污染物和其他有毒有害物质[2], 因此必 须污泥进行相应的处理。首先需要对污泥进行浓缩, 为之 后污泥的存储、运输与污泥最终处置创造更好更有利的条 件[3]。然后对污泥进行调理, 改善污泥的相关性质, 并且 为后面的污泥脱水提供有效帮助[4]。也可以采用卫生填埋 的方法, 但是卫生填埋对于环境的影响比较大, 因此对污 泥的卫生填管理应该非常严格 [5]。

污泥过程减量: 污泥过程减量包括细胞溶解和隐性增 长、解偶联作用和微生物的捕食作用。

细胞溶解和隐性增长: 微生物利用细菌残存细胞物质 作为营养物质重新进行代谢称为细胞溶解隐性增长。其中 细菌细胞溶解是其中的限制步骤[6]。目前利用机械(物理) 方法形成细菌溶胞简单灵活而且高效[7], 但是能量耗费较 大。采用化学方法以 $\mathrm{O}_{3} 、 \mathrm{ClO}_{2} 、 \mathrm{H}_{2} \mathrm{O}_{2}$ 等强氧化剂氧化的方 法 $[8,9]$, 该方法破解效率高, 不产生有害副产物, 但是处
理成本高, 出水水质差, 特别是出水中 $\mathrm{N} 、 \mathrm{P}$ 含量高, 因 此需要进一步优化。

解偶联作用: 微生物的合成代谢和分解代谢是通过呼吸 速率紧密耦合在一起, 通过某些方法, 例如重金属、过剩能 量基团、特殊温度、营养物质受到限制等条件。能量就会发 生溢散, 从而达到污泥减量的效果。解偶联剂实际操作简单, 且实际的使用过程中间发现其会改变污泥的沉降性能 [10], 更有甚者可能会导致污泥膨胀[11]。哈尔滨工业大学的王晓 东利用解偶联剂THPS(四羟甲基硫酸磷)实现污泥减量, 发现 当 THPS 的浓度为 $3 \mathrm{mg} / \mathrm{L}$ 的时候, 污泥减量的效果达到了 $39.1 \%$, 在这种工况条件下，对COD、氨氮、总氮和总磷的 平均去除率分别 $83.1 \% 、 86.4 \% 、 69.8 \%$ 和 $58.9 \%[12]$ 。

\section{2. 实验材料和方法}

实验采用六组 $\mathrm{SBR}$ 工艺, $\mathrm{SBR}$ 的有效容积为 $4 \mathrm{~L}$ 。每天 运行两个周期: 缺氧搅拌 $3 \mathrm{~h}$, 好氧曝气 $6 \mathrm{~h}$, 静置沉淀 $3 \mathrm{~h}$ 。 每次换水 $2 \mathrm{~L}$, 每天排泥 $300 \mathrm{ml}$, 溶解氧浓度维持在 2 $5 \mathrm{mg} / \mathrm{L}$ ，系统的MLSS(混合液活性污泥浓度)维持在 1000 $2500 \mathrm{mg} / \mathrm{L}$ 。实验采用的是模拟生活污水的人工配水，相应 的进水组成如下:

表1 实验的配水组成。

\begin{tabular}{llll}
\hline 水处理指标 & 指标浓度 $(\mathbf{m g} / \mathbf{L})$ & 物质名称 & 物质投加量 $(\mathbf{m g} / \mathbf{L})$ \\
\hline $\mathrm{COD}$ & $300 \mathrm{mg} / \mathrm{L}$ & 葡萄糖 & 281 \\
$\mathrm{NH}_{4}{ }^{+}-\mathrm{N}$ & $30 \mathrm{mg} / \mathrm{L}$ & $\mathrm{NH}_{4} \mathrm{Cl}$ & 115 \\
$\mathrm{PO}_{4}{ }^{3-}-\mathrm{P}$ & $5 \sim 8 \mathrm{mg} / \mathrm{L}$ & $\mathrm{KH}_{2} \mathrm{PO}_{4}$ & $22 \sim 35$ \\
无机盐 & & 无水CaCl & 10 \\
无机盐 & & $\mathrm{MgCl}_{2}$ & 60 \\
碱度 & & 小苏打 & 300 \\
\hline
\end{tabular}

微生物的生长需要适量的微量元素, 在配水中间每升进水加入 $1 \mathrm{ml}$ 的微量元素溶液。微量元素溶液的组成如下表:

表2 微生物的微量元素溶液。

\begin{tabular}{llll}
\hline 物质 & 浓度 $(\mathbf{g} / \mathbf{l})$ & 物质 & 浓度 $(\mathbf{g} / \mathbf{l})$ \\
\hline $\mathrm{ZnSO} 4$ & 0.15 & $\mathrm{CoCl} 2$ & 0.15 \\
$\mathrm{CuSO} 4$ & 0.06 & 碘化钾 & 0.18 \\
$\mathrm{FeCl} 3$ & 1.5 & $\mathrm{H} 3 \mathrm{BO} 3$ & 0.06 \\
$\mathrm{NaMoO} 4$ & 0.15 & EDTA & 5 \\
\hline
\end{tabular}

实验采用尼泊金丙酯这种解偶联剂来研究其对 SBR 系统的污水处理和污泥减量的效果。

尼泊金丙酯又名对羟基苯甲酸丙酯, 对羟基安息香酸 丙酯。无色小结晶或白色粉末,尼泊金丙酯在食品、药品、 化妆品、皮革以及塑料制品等的抑菌防腐与防霉方面应用 十分广泛。

试验中选择 6 个不同的尼泊金甲酯浓度梯度，分别为 $0 \mathrm{mg} / 1$ （空白）、 $1 \mathrm{mg} / 1 、 5 \mathrm{mg} / 1 、 10 \mathrm{mg} / 1 、 30 \mathrm{mg} / 1 、 50 \mathrm{mg} / 1$ 。 对应的反应器分别为R $0 、 R 1 、 R 2 、 R 3 、 R 4 、 R 5$ 。进水对 应的指标用influx表示。检测的污水指标和污泥指标以及 相应的检测方法如下:

表3 实验的检测指标及方法。

\begin{tabular}{ll}
\hline 检测指标 & 检测方法 \\
\hline $\mathrm{COD}$ & 重铬酸盐法 \\
氨氮 & 纳氏试剂分光光度法 \\
总氮 & 总有机碳总氮分析仪TC-VCPN \\
磷酸盐 & 钿锑抗分光光度法 \\
$\mathrm{SV}_{30}$ & $30 \mathrm{~min}$ 静沉法 \\
$\mathrm{MLSS}$ & 重量法 \\
\hline
\end{tabular}




\section{3. 尼泊金丙酯对系统污泥减量和污水处理的影} 响

\section{1. 尼泊金丙酯对系统污泥减量的影响}

\subsection{1. 尼泊金丙酯对污泥累计增长量的影响}

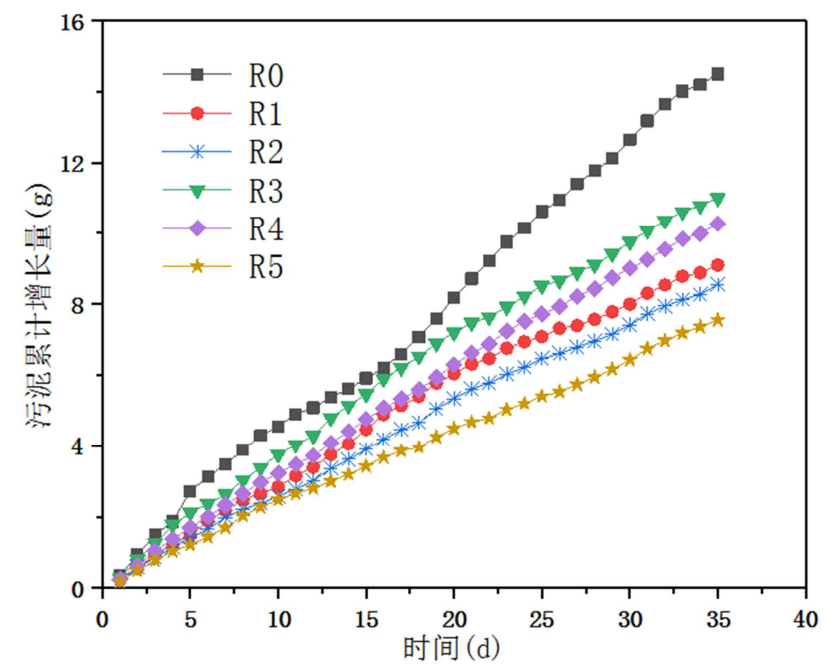

图1 每个反应器的污泥累计增长量。

从图上可以看出在最初的 5 天时间内, 每个反应器的 污泥累计增长量相对来说差别不大, 说明在最初的五天内,
污泥表观增长率基本一样, 说明经过驯化后的活性污泥活 性相差不大。随着运行时间的不断增加, R0首先呈现出相 对比较明显的趋势, 表观产率相对来说比较高。运行 10 多天每个反应器的污泥累计增长量才出现比较显著的差 别, 在实验运行的 35 天的时间内, 每个反应器的污泥累计 增长量为: $R 0=14.502 \mathrm{~g}, \mathrm{R} 1=9.105 \mathrm{~g}, \mathrm{R} 2=8.545 \mathrm{~g}$, $\mathrm{R} 3=11.001 \mathrm{~g}, \mathrm{R} 4=10.278 \mathrm{~g}, \mathrm{R} 5=7.5585 \mathrm{~g}$ 。经过计算各个反 应器的减量效果为 $R 1=37 \%, R 2=41 \%, R 3=24 \%, R 4=29 \%$, $\mathrm{R} 5=47 \%$ 。在进水的浓度为 $30 \mathrm{mg} / \mathrm{L}$ 的时候, 污泥的减量效 果达到最好。

当进水中间的尼泊金丙酯的浓度为 $30 \mathrm{mg} / \mathrm{L}$ 的时候, 污 泥减量的效果最好为 $47 \%$ 。

\subsection{2. 尼泊金丙酯对污泥表观产率的影响}

污泥表观产率系数Yobs 是实际测定的污泥产量与有 机基质降解量的比值, 即降解单位有机基质所增长的污泥 量, 是表观污泥产量的重要指标之一。由于实验中 $\mathrm{R} 0$ 至 R5 各组反应器中对有机基质的去除效率均不相同, 为了刨 除各个反应器有机基质降解量差异对污泥减量效果的影 响, 因此需要研究污泥的表观产率系数Yobs:

$$
Y_{o b s}=\frac{\text { 污泥累计增长量 }}{C O D \text { 累计消耗量 }}
$$

每个反应器的COD累计消耗量如下表所示:

表4 每个反应器的COD的累计增长量。

\begin{tabular}{lllllll}
\hline 反应器 & R0 & R1 & R2 & R3 & R4 & R5 \\
\hline COD累计消耗量 $(\mathrm{g})$ & 38.6 & 38.3 & 39.8 & 38.8 & 38.8 & 39.1 \\
\hline
\end{tabular}

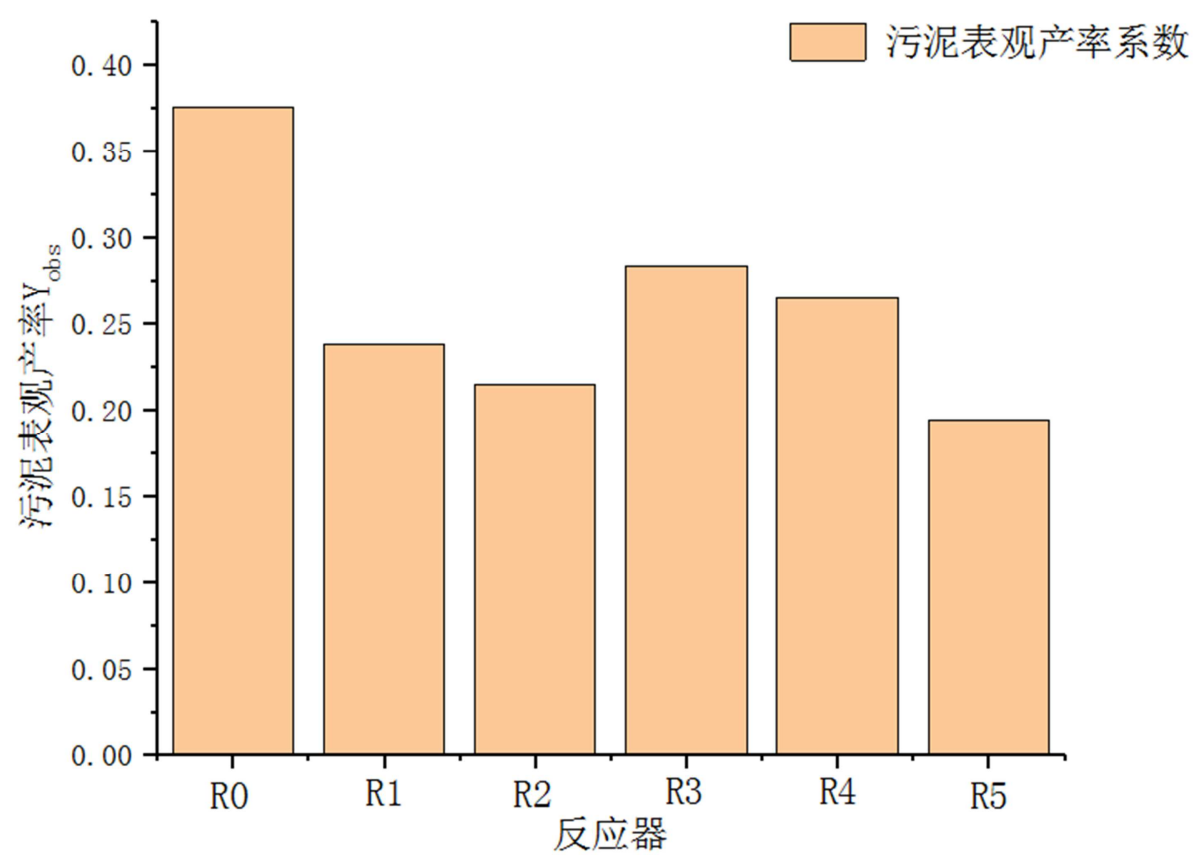

图2 每个反应器的污泥表观产率系数 $\mathrm{Y}_{\mathrm{obs}}$ 。

经过计算每个反应器的 $Y_{\text {obs }}$ 分别为: $Y_{\text {obs }}(R 0)=0.37$, $\mathrm{Y}_{\mathrm{obs}}(\mathrm{R} 1)=0.23, \mathrm{Y}_{\mathrm{obs}}(\mathrm{R} 2)=0.21, \mathrm{Y}_{\mathrm{obs}}(\mathrm{R} 3)=0.28, \mathrm{Y}_{\mathrm{obs}}(\mathrm{R} 4)=0.26$, $Y_{\text {obs }}(R 5)=0.19$ 。 空白组的 $Y_{\text {obs }}$ 最大为 0.37 。说明空白组的污
泥活性最好, 对于污染物的代谢和去除正常。污泥表观产 率最高, 其余的每个反应器的污泥表观产率都比空白组低, 
说明其余的反应器都具有污泥减量的效果。但是R5的污泥 表观产率最低, 说明污泥减量的效果最好。

从污泥表观产率系数可以看到, 空白组的污泥表观产 率系数最高, 说明尼泊金丙酯确实具有污泥减量的效果,
并且投加不同浓度的尼泊金丙酯对于系统污泥减量的效 果也会有所不同。当尼泊金丙酯的浓度为 $30 \mathrm{mg} / \mathrm{L}$ 的时候, 污泥减量的效果最好。

\subsection{3. 尼泊金丙酯对污泥沉降性能的影响}
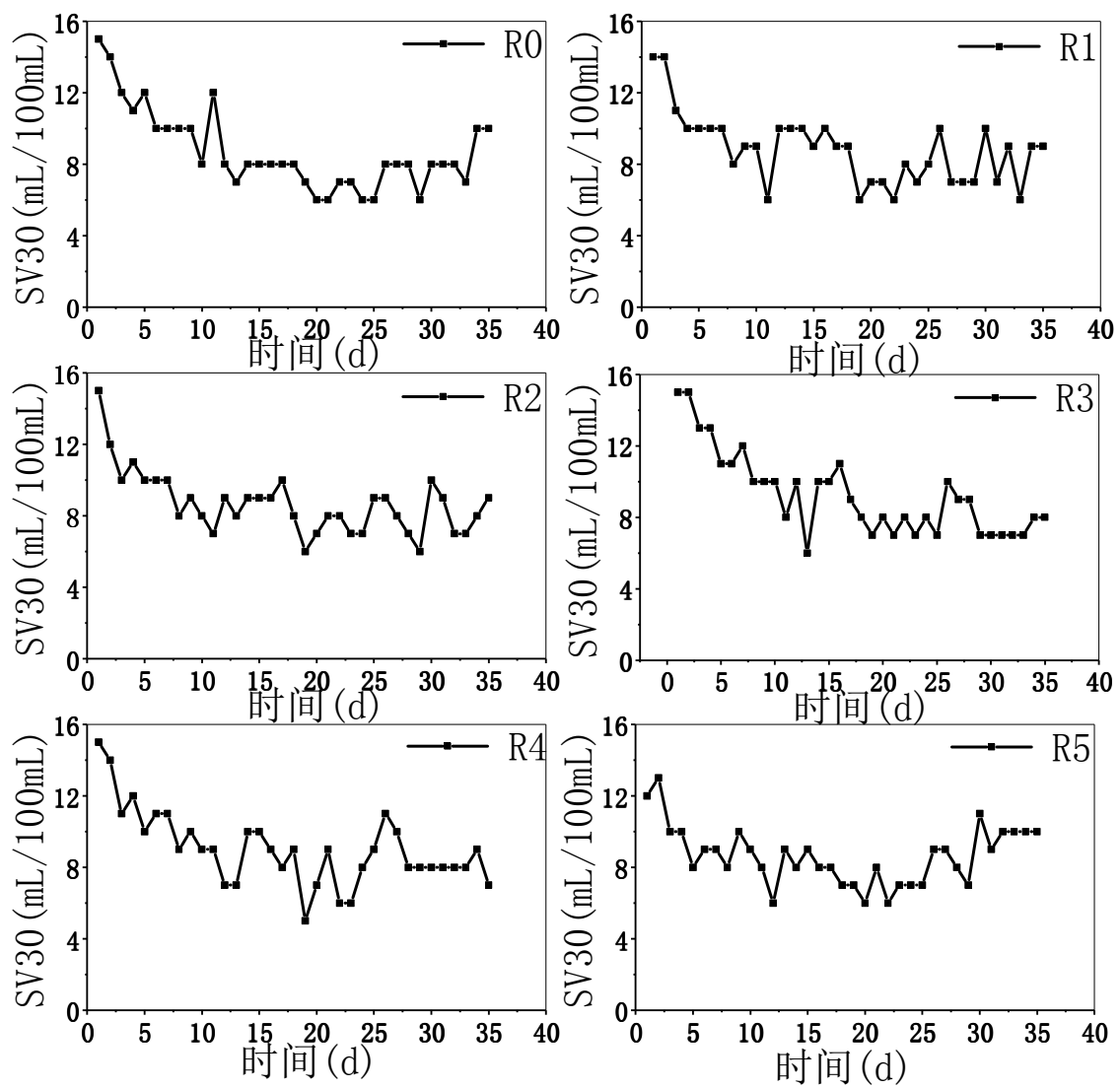

图3 每个反应器的SV30。

从图上可以看出 $\mathrm{R} 0$ 的 SV 30 从最初的 $16 \%$ 不断下降到 $8 \%$, 然后 SV 30 相对来说比较稳定, 一直维持在 $8 \%$ 左右。 $\mathrm{R} 1$ 从最初的 $14 \%$ 下降到 $8 \%$, 然后 SV 30 相对来说比较稳定, 基本也是稳定在 $8 \%$ 左右。 $\mathrm{R} 2$ 的 SV 30 从最初的 $16 \%$ 下降到 $8 \%$ 之后, 波动比较小, 相对来说比较稳定。R3的SV30的 变化规律和 $\mathrm{R} 2$ 比较接近。 $\mathrm{R} 4$ 与 $\mathrm{R} 5$ 到 $\mathrm{SV} 30$ 的变化规律也是 比较接近的。可以看到, 每个反应器在最初的一段时间 SV30都会有所下降, 说明加入尼泊金甲酯有利于系统污 泥的沉降。经过一段时间的调整系统的SV30相对来说比 较稳定, 系统的沉降性能又得到逐渐的恢复。经过计算每 个反应器的 $\mathrm{SV} 30$ 的平均值为: $\mathrm{A}(\mathrm{R} 0)=8.71 \pm 2.25(\%)$, $\mathrm{A}(\mathrm{R} 1)=8.8 \pm 1.95(\%)$ $\mathrm{A}(\mathrm{R} 2)=8.68 \pm 1.76(\%)$, $\mathrm{A}(\mathrm{R} 3)=9.22 \pm 2.31(\%)$, $\mathrm{A}(\mathrm{R} 5)=8.62 \pm 1.62(\%)$ 。 $\mathrm{A}(\mathrm{R} 4)=9.02 \pm 2.09(\%)$,

结论: R0与 R1的SV30相差不大, 说明一定量的抑菌 剂尼泊金丙酯对于系统的SV30的影响不是特别大。但是 $\mathrm{R} 3$ 与 $\mathrm{R} 4$ 的沉降性相对来说与 $\mathrm{R} 0$ 差别较大。污泥减量最好 的条件下, SV30的平均值和空白组基本没有什么差别。
从图上可以看出, 空白组的SVI在前面的几天波动相 对来说比较大, 之后的一段时间相对来说比较稳定, 在第 9 天的时候SVI的值达到了 160 , 可能出现了污泥的膨胀。 在第15 22天SVI的值相对来说比较低, 可能的原因是这 段时间污泥的无机质相对来说比较多, 污泥可你比较细。 $\mathrm{R} 1, \mathrm{R} 2, \mathrm{R} 3, \mathrm{R} 4$ 的变化规律大致相似。从最后几天的变 化规律来看, $\mathrm{R} 0$ 与 $\mathrm{R} 5$ 还是有一定的差别。 $\mathrm{R} 5$ 最后几天的 平均值相对于 $\mathrm{R} 0$ 最后几天的平均值应该低 10 左右。从图上 可以看出每个反应器的SVI值有较大的波动, 但是波动的 范围基本都还在 $50 \sim 150$ 之间说明污泥的沉降性能相对来 说比较良好。经过计算每个反应器的 SVI 值: $\mathrm{A}(\mathrm{R} 0)=78.61 \pm 27.03 \quad, \quad \mathrm{~A}(\mathrm{R} 1)=91.45 \pm 28.35$ $\mathrm{A}(\mathrm{R} 2)=97.79 \pm 26.18 \quad, \quad \mathrm{~A}(\mathrm{R} 3)=89.20 \pm 26.57$ $\mathrm{A}(\mathrm{R} 4)=102.19 \pm 26.13, \mathrm{~A}(\mathrm{R} 5)=96.37 \pm 29.24$ 。加入尼泊金丙 酯会提高系统的SVI值。

从图上可以看到每个反应器的SVI基本都在 $50 \sim 150$ 之间, 说明污泥的沉降性能正常。而且加入尼泊金丙酯会 提高SBR系统的SVI的值。 

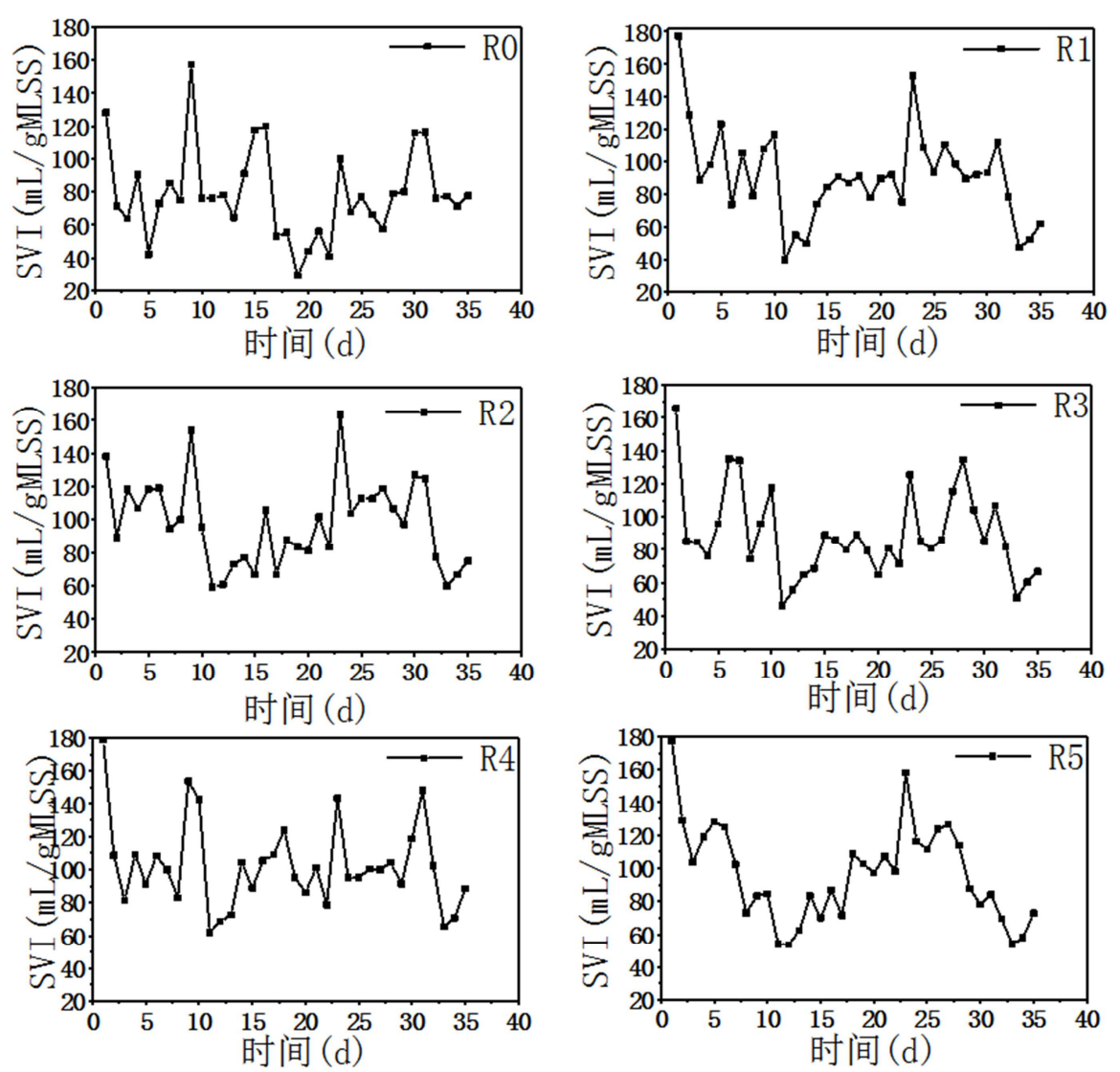

图4 每个反应器的SVI。

3.2. 尼泊金丙酯对系统污水处理的影响效果

\subsection{1. 尼泊金丙酯对系统COD的影响}

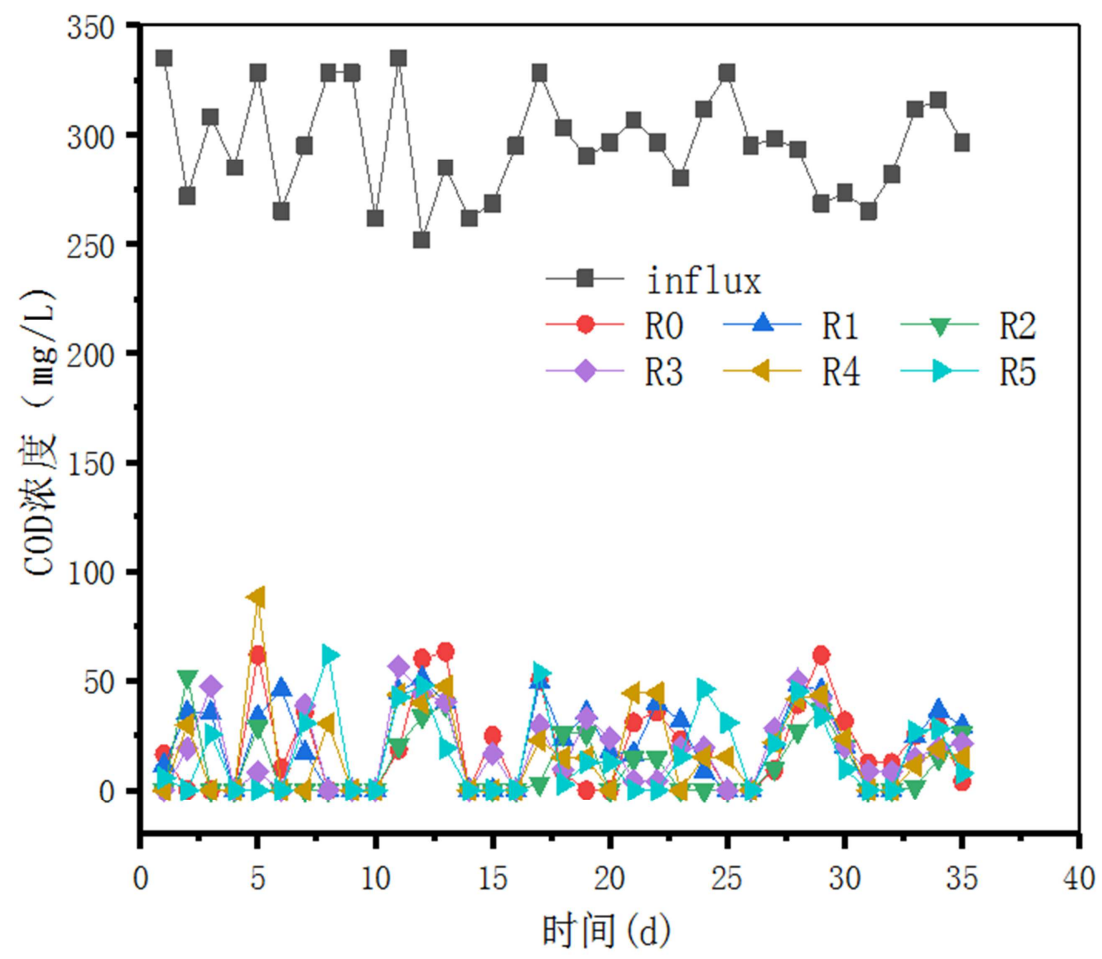

图5 进水和每个反应器的COD的浓度。 

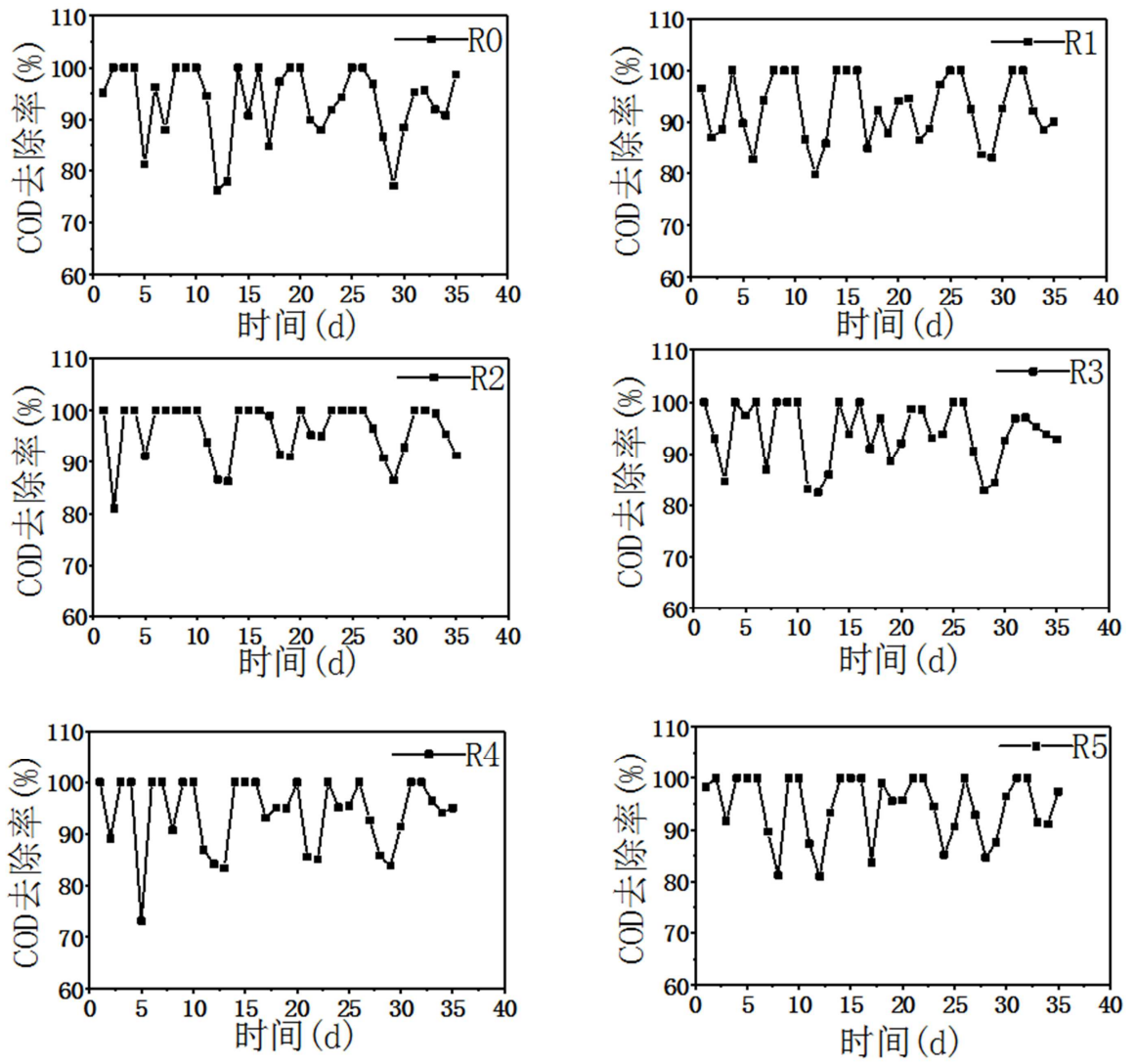

图6 每个反应器的COD的去除率。

从图上可以看出，投加抑制剂尼泊金丙酯之后，系统 的COD的去除率一般会有所下降,之后 COD的去除率会逐 渐上升, 说明生物处理系统具有一定的抗冲击负荷。在整 个实验的过程中间 $\mathrm{COD}$ 的去除效果良好, 基本都在 $80 \%$ 以 上。经过计算每个反应器的 $\mathrm{COD}$ 的平均去除率为: $\mathrm{A}(\mathrm{R} 0)=93.35 \pm 7.24(\%), \mathrm{A}(\mathrm{R} 1)=92.56 \pm 6.35(\%), \mathrm{A}(\mathrm{R} 2)=96.11$ $\pm 5.25(\%), \mathrm{A}(\mathrm{R} 3)=93.89 \pm 5.86(\%), \mathrm{A}(\mathrm{R} 4)=94.02 \pm 6.88(\%), \mathrm{A}(\mathrm{R}$ $5)=94.53 \pm 6.17(\%)$ 。从 COD的平均去除率可以看到尼泊金 丙酯对于系统的COD的去除率没有什么影响, 可能的原因 是 $\mathrm{COD}$ 是多种微生物的碳源, 导致整体上COD的去除率变 化不大。

\subsection{2. 尼泊金丙酯对系统 $\mathrm{NH}_{4}{ }^{+}-\mathrm{N}$ 的影响}

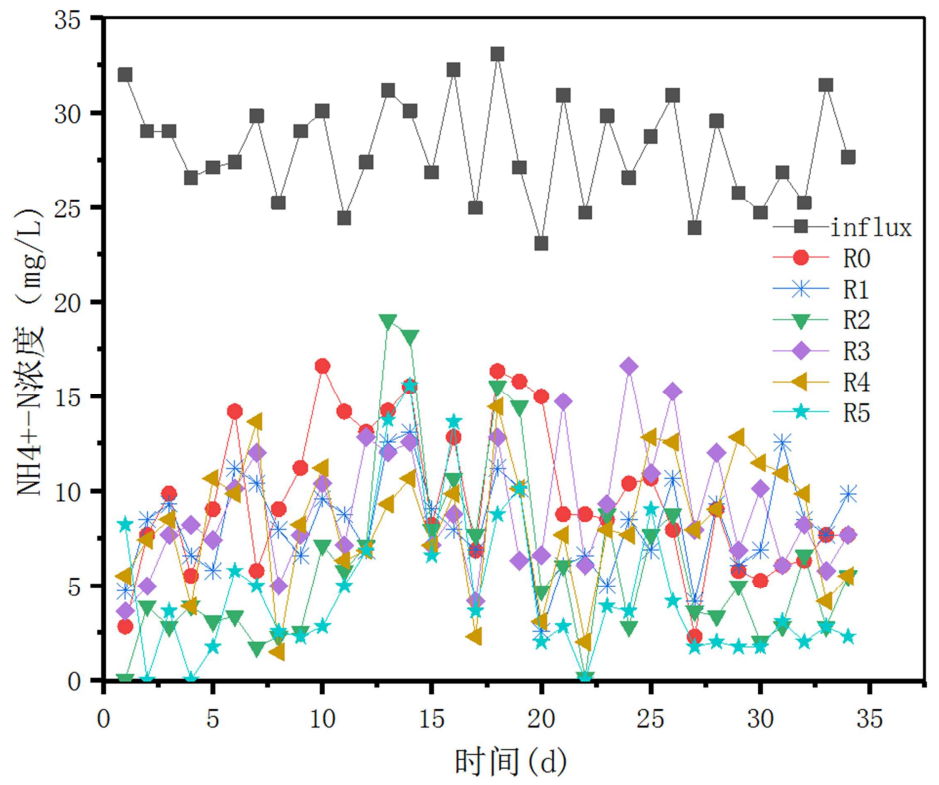

图7 进水和每个反应器的 $\mathrm{NH}_{4}{ }^{+}-\mathrm{N}$ 浓度。 
从图上可以看出进水的 $\mathrm{NH}_{4}{ }^{+}-\mathrm{N}$ 浓度基本都在 $30 \mathrm{mg} / 1$ 左右波动, $\mathrm{R} 0$ 在最初的九天出水的 $\mathrm{NH}_{4}{ }^{+}-\mathrm{N}$ 的浓度 相对来说比较低, 基本都在 $8 \mathrm{mg} / 1$ 左右 $\mathrm{NH}_{4}{ }^{+}-\mathrm{N}$ 的去除率 基本都在 $60 \%$ 以上。在第 $10 \sim 20$ 天 $\mathrm{NH}_{4}{ }^{+}-\mathrm{N}$ 浓度相对来说 比较高, 而且去除率比较不稳定, 都在 $60 \%$ 以下, 在 20 天之后 $\mathrm{NH}_{4}{ }^{+}-\mathrm{N}$ 的浓度基本都维持在 $8 \mathrm{mg} / 1$ 之下。 $\mathrm{R} 1$ 出水 的 $\mathrm{NH}_{4}{ }^{+}-\mathrm{N}$ 浓度相对来说比较稳定, 都在 $4 \sim 12 \mathrm{mg} / \mathrm{l}$ 之间, $\mathrm{NH}_{4}{ }^{+}-\mathrm{N}$ 的去除率都在 $60 \%$ 以上。 $\mathrm{R} 2$ 在前 12 天和 20 天之后 出水的 $\mathrm{NH}_{4}{ }^{+}-\mathrm{N}$ 浓度相对来说比较低, 但是在 $12 \sim 20$ 天之 间出水的 $\mathrm{NH}_{4}{ }^{+}-\mathrm{N}$ 浓度相对来说比较高, 这段时间 $\mathrm{NH}_{4}{ }^{+}-\mathrm{N}$ 的去除率都在 $60 \%$ 以下。从整体上看 $\mathrm{R} 3$ 的出水 $\mathrm{NH}_{4}{ }^{+}-\mathrm{N}$ 浓度相对来说比较稳定, 但是出水的 $\mathrm{NH}_{4}{ }^{+}-\mathrm{N}$ 平 均浓度比 $\mathrm{R} 2$ 的浓度要高, $\mathrm{NH}_{4}{ }^{+}-\mathrm{N}$ 的去除率都在 $50 \%$ 以上。
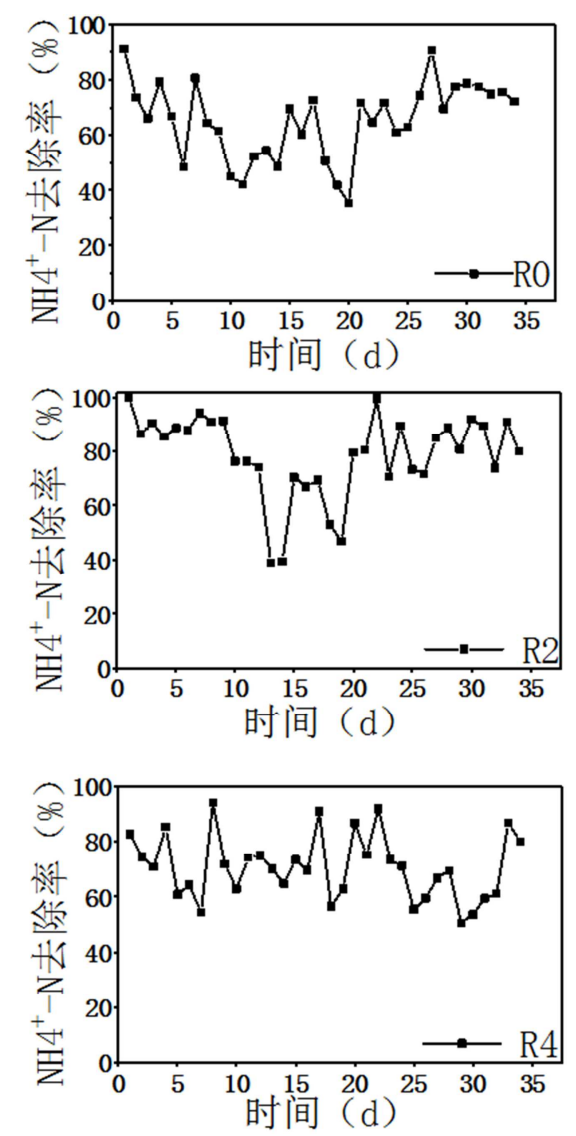

$\mathrm{R} 3$ 在最初的 4 天出水的 $\mathrm{NH}_{4}{ }^{+}-\mathrm{N}$ 浓度比较低, 之后的 $\mathrm{NH}_{4}{ }^{+}-\mathrm{N}$ 浓度一直都是比较高的。 $\mathrm{R} 5$ 在前 12 天和 26 天之 后出水的 $\mathrm{NH}_{4}{ }^{+}-\mathrm{N}$ 浓度相对来说比较低, 去除率比较良好。 12 26天出水的 $\mathrm{NH}_{4}{ }^{+}-\mathrm{N}$ 浓度相对来说比较高。经过计算 每 个系统 $\mathrm{NH}_{4}{ }^{+} \mathrm{-N}$ 的平均去除率为: $\mathrm{A}(\mathrm{R} 0)=65.47 \pm 13.86(\%) \quad, \quad \mathrm{A}(\mathrm{R} 1)=70.89 \pm 8.26(\%) \quad$ ， $\mathrm{A}(\mathrm{R} 2)=78.59 \pm 15.42(\%) \quad, \quad \mathrm{A}(\mathrm{R} 3)=68.13 \pm 10.72(\%) \quad$, $\mathrm{A}(\mathrm{R} 4)=70.55 \pm 11.72(\%), \mathrm{A}(\mathrm{R} 5)=83.84 \pm 12.91(\%)$ 。可以 看到加入尼泊金丙酯这种抑菌剂对于系统 $\mathrm{NH}_{4}{ }^{+}-\mathrm{N}$ 的平 均去除率有所提高, 可能的原因是尼泊金丙酯抑制了与 硝化细菌形成竞争关系的微生物, 使得硝化细菌相对占 据优势, 提高了 $\mathrm{NH}_{4}{ }^{+}-\mathrm{N}$ 的的去除率。但是总体的 $\mathrm{NH}_{4}{ }^{+}-\mathrm{N}$ 去除率偏低, 主要是温度的影响。
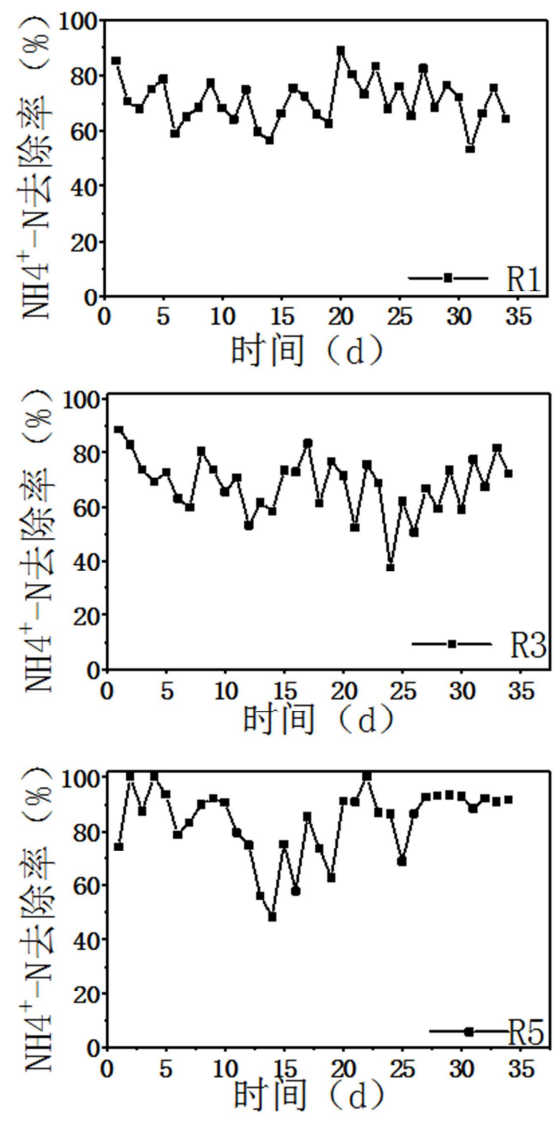

图8 每个反应器的 $\mathrm{H}_{4}{ }^{+}-\mathrm{N}$ 的去除率。

\subsection{3. 尼泊金丙酯对系统 $\mathrm{PO}_{4}{ }^{3-}-\mathrm{P}$ 的影响}

从图上可以看出 R1, R2, R3, R4这五个反应器的 $\mathrm{PO}_{4}{ }^{3-}-\mathrm{P}$ 的去除率都是经过了先下降, 然后不断上升, 最 后保持稳定的过程。说明加入丙酯对于系统与 $\mathrm{PO}_{4}{ }^{3-}-\mathrm{P}$ 的微 生物有显著影响, 但是微生物具有一定的适应能力, 活性 逐渐得到恢复, 使得系统的 $\mathrm{PO}_{4}{ }^{3-}-\mathrm{P}$ 的去除率有所上升, 最
后系统保持相对稳定的状态, 但是R6的磷酸盐的去除规律 与前面几个反应器都不相同, 需要深入研究规律。经过计 算系统 $\mathrm{PO}_{4}{ }^{3-}-\mathrm{P}$ 的平均去除率为: $\mathrm{A}(\mathrm{R} 0)=58.47 \pm 21.23(\%)$, $\mathrm{A}(\mathrm{R} 1)=56.48 \pm 16.03(\%), \mathrm{A}(\mathrm{R} 2)=52.47 \pm 19.43(\%), \mathrm{A}(\mathrm{R} 3)=57.0$ $9 \pm 20.81(\%), \mathrm{A}(\mathrm{R} 4)=55.23 \pm 14.79(\%), \mathrm{A}(\mathrm{R} 5)=67.36 \pm 16.05(\%)$ 。 


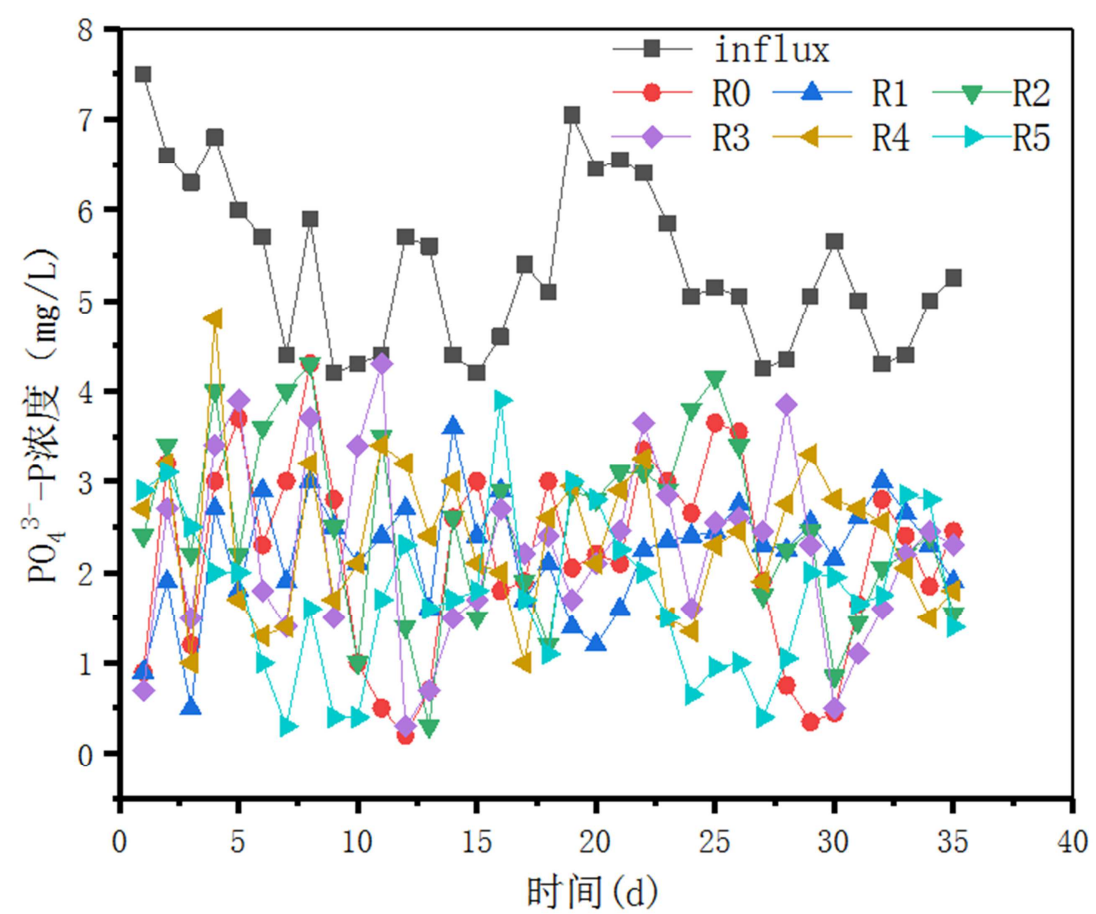

图9 进水和每个反应器的 $\mathrm{PO}_{4}{ }^{3-}-\mathrm{P}$ 浓度。
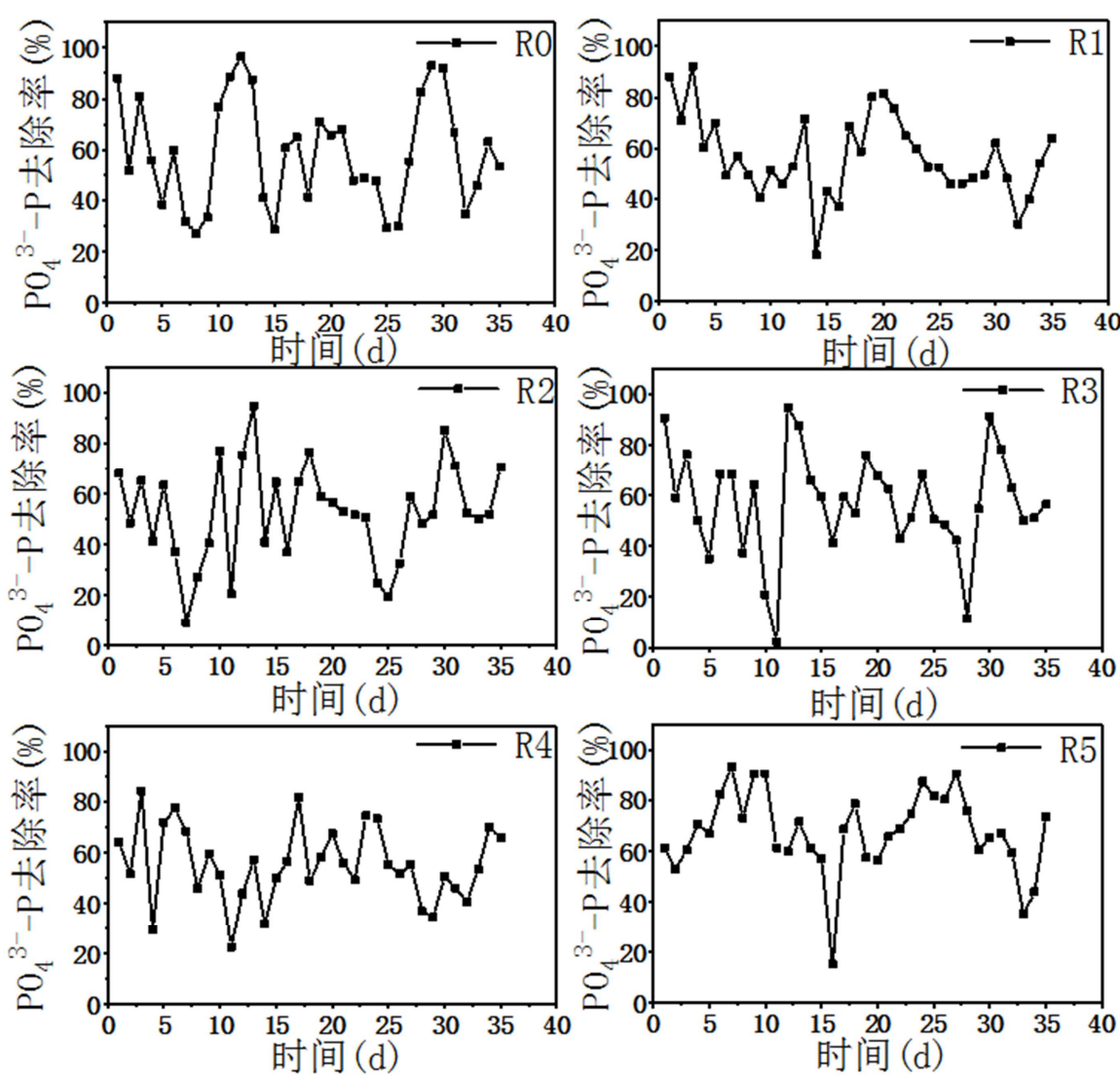

图10 每个反应器的 $\mathrm{PO}_{4}{ }^{3-} \mathrm{P}$ 的去除率。

\section{4. 结论}

当进水中间加入 $30 \mathrm{mg} / \mathrm{L}$ 的尼泊金丙酯的时候, 污泥的 减量效果最佳达到了 $47 \%$ 。此条件下, $\mathrm{COD}$ 的平均去除
率由空白组的 $93.35 \%$ 提高到 $94.53 \% ; \mathrm{NH}_{4}{ }^{+} \mathrm{N}$ 的平均去除 率由空白组的 $65.47 \%$ 提高到 $83.84 \%$; $\mathrm{PO}_{4}{ }^{3-}-\mathrm{P}$ 的平均去除率 会从空白组的 $58.47 \%$ 提高到 $67.36 \%$; 在水处理工程中, 污 泥保持良好的沉降性能。 


\section{参考文献}

[1] 蒋一凡.SBR/OSA工艺污泥减量机理的研究 [D].扬州大 学,2017。

[2] 李鸿江, 顾莹莹, 赵由才.污泥资源化利用技术[M].北京: 冶金工业出版社，2010：31-37。

[3] 朱开金, 马忠亮.污泥处理技术及资源化利用[M]. 北京: 化 学工业出版社, 2006：126-129。

[4] 王绍文, 秦华.城市污泥资源利用与污水土地处理技术 $[\mathrm{M}]$. 北京：中国建筑工业出版社，2007：52-56。

[5] 翁焕新.污泥无害化、减量化、资源化处理新技术 $[\mathrm{M}]$. 北京: 科学出版社, 2009: 11-72。

[6] 冯骁驰.污泥过程减量的复合解偶联剂特性及对处理效能 影响研究[D].哈尔滨工业大学,2013。

[7] 邵尤炼, 缪恒锋,任洪艳,赵明星,阮文权.胞外多聚物在厌氧 污泥颗粒化成核过程中的特性 [J]. 环境工程学 报,2014,8(07):2697-2702。

[8] 吴俊锋,冯晓西,周益.一种基于隐性生长工艺的污泥减量化 工艺及装置[P].上海：CN101704627A,2010-05-12。

[9] 王慧荣,梅荣武,韦彦斐,李明智,张宇,任旭峰。一种用于污泥 原位减量的微生物菌剂及其制备方法和应用[P]. 浙江: CN104789504A,2015-07-22。

[10] 仓一华,吴敏,赵海云,等.活性污泥法污水处理系统好氧单元 剩余污泥减量的方法 [P]. 中国，发明专 利,CN102145972A,2011。

[11] 陈志英.解偶联代谢用于污泥减量化的研究 [D]. 同济大 学,2006。

[12] 王晓东.THPS对间歇式IFAS的污泥减量特性及工艺运行的 影响[D].哈尔滨工业大学,2017。 